\title{
Informações toxicológicas de alguns fitoterápicos utilizados no Brasil
}

\author{
Monica Silva dos Reis Turolla, Elizabeth de Souza Nascimento*
}

Departamento de Análises Clínicas e Toxicológicas, Faculdade de Ciências Farmacêuticas, Universidade de São Paulo

*Correspondência:

E.S. Nascimento

Departamento de Análises Clínicas e

Toxicológicas

Faculdade de Ciências Farmacêuticas da Universidade de São Paulo

Av Lineu Prestes, 580, bloco 13B 05389-970 - São Paulo, SP - Brasil

E-mail: esnasci@usp.br
As plantas medicinais vêm sendo utilizadas com finalidades terapêuticas há milhares de anos. Seu uso popular foi propagado de geração em geração e descrito nas diversas farmacopéias. A partir do desenvolvimento da química orgânica, tornou-se possivel obter substâncias puras através do isolamento de princípios ativos de plantas, entre elas, a digoxina e a morfina, resultando em desinteresse pela pesquisa de substâncias de origem vegetal. Entretanto, a partir da década de 1980, foram desenvolvidos novos métodos de isolamento de substâncias ativas, tornando-se possivel identificar substâncias em amostras complexas como os extratos vegetais, ressurgindo o interesse por compostos de origem vegetal que pudessem ser utilizados como protótipos para o desenvolvimento de novos fármacos. Atualmente, apesar da crescente importância dos medicamentos fitoterápicos, relativamente poucos estudos foram realizados a fim de comprovar sua eficácia e segurança, sendo que muitas plantas ainda são utilizadas com base somente no seu uso popular bem estabelecido. O objetivo deste trabalho foi avaliar, junto aos principais bancos de dados e fontes públicas de informação, a disponibilidade de dados de toxicidade pré-clínica de dez plantas medicinais comercializadas na forma de medicamentos fitoterápicos no Brasil,. Observou-se que há poucos dados sobre a toxicidade pré-clínica das dez plantas pesquisadas, mas, de maneira geral, os experimentos em animais demonstraram baixa toxicidade aguda, subaguda e crônica e não mostraram atividades mutagênicas ou teratogênicas.
Unitermos

- Informações toxicológicas

- Medicamentos fitoterápicos

- Plantas medicinais

- Toxicidade pré-clínica

\section{INTRODUÇÃO}

Há milhares de anos, o homem vem utilizando os recursos da flora no tratamento de diversas patologias. Há relatos, por exemplo, do uso de plantas com finalidades terapêuticas por volta de 3.000 a.C. na obra Pen $T s^{\prime}$ 'ao do chinês Shen Nung (Ko, 1999; Tyler, 1996). No ano
78 d.C., o botânico grego Pedanios Dioscorides descreveu cerca de 600 plantas medicinais, além de produtos minerais e animais no tratado De Materia Medica. Este tratado permaneceu como fonte de referência por mais de catorze séculos (Robbers, Speedie, Tyler, 1996; Tyler, 1996). Foi através da observação e da experimentação pelos povos primitivos que as propriedades terapêuticas de deter- 
minadas plantas foram sendo descobertas e propagadas de geração em geração, fazendo parte da cultura popular.

No século XVI, o médico suíço Philippus Aureolus Theophrastus Bombastus von Hohenheim, conhecido como Paracelsus (1493-1541), formulou a "Teoria das Assinaturas", baseada no provérbio latim similia similibus curantur, "semelhante cura semelhante". Com esta teoria acreditava-se que a forma, a cor, o sabor e o odor das plantas estavam relacionados com as suas propriedades terapêuticas, podendo dar indícios de seu uso clínico. Algumas destas plantas passaram a fazer parte das farmacopéias alopáticas e homeopáticas a partir do século XIX, quando se começou a investigar suas bases terapêuticas (Elvin-Lewis, 2001).

$\mathrm{O}$ isolamento da morfina da Papaver somniferum em 1803 pelo farmacêutico Friedrich Wilhelm Adam Sertürner, marcou o início do processo de extração de princípios ativos de plantas. A partir de então, outras substâncias foram isoladas, como por exemplo, a quinina e a quinidina obtidas da Cinchona spp, em 1819, e a atropina da Atropa belladona, em 1831, que passaram a ser utilizadas em substituição aos extratos vegetais (Schulz, Hänsel, Tyler, 2001; Tyler 1996).

Assim, a produção de fármacos via síntese química, o crescimento do poder econômico das indústrias farmacêuticas e a ausência de comprovações científicas de eficácia das substâncias de origem vegetal aliada às dificuldades de controle químico, físico-químico, farmacológico e toxicológico dos extratos vegetais até então utilizados, impulsionaram a substituição destes por fármacos sintéticos (Rates, 2001).

Após a década de 1960, observou-se, então, um desinteresse da indústria farmacêutica e dos institutos de pesquisa pela busca de novas substâncias de origem vegetal, por se acreditar que já haviam sido isoladas as principais substâncias ativas das drogas vegetais conhecidas, bem como já haviam sido realizadas todas as possíveis modificações químicas de interesse destas substâncias (Schenckel, Gosman, Petrovick, 2000).

Entretanto, a partir dos anos 1980, os avanços técnicos e o desenvolvimento de novos métodos de isolamento de substâncias ativas a partir de fontes naturais, permitiram maior rapidez na identificação de substâncias em amostras complexas como os extratos vegetais, ressurgindo o interesse pela pesquisa destas substâncias como protótipos para o desenvolvimento de novos fármacos.

Assim, mesmo com o desenvolvimento de grandes laboratórios farmacêuticos e dos fármacos sintéticos, as plantas medicinais permaneceram como forma alternativa de tratamento em várias partes do mundo. Observou-se nas últimas décadas a revalorização do emprego de preparações fitoterápicas. Assim, alguns grupos farmacêuticos passaram a desenvolver esforços voltados para o aprimo- ramento de medicamentos fitoterápicos e sua produção em escala industrial. O novo avanço dos medicamentos fitoterápicos, longe de ser volta ao passado, caracteriza-se pela busca de produção em escala industrial, diferentemente das formas artesanais que caracterizaram os estágios iniciais de sua utilização.

Atualmente, os fitoterápicos são amplamente utilizados em diversos países. Na África, por exemplo, $80 \%$ da população depende do uso destes medicamentos, os quais representam alternativa frente ao alto custo dos fármacos sintéticos. $\mathrm{O}$ mercado mundial de medicamentos fitoterápicos é de US\$ 43 bilhões por ano. Somente nos Estados Unidos da América, este mercado representa US\$ 5 bilhões por ano, sendo o setor de mais rápido crescimento no mercado farmacêutico norte-americano (Aschwanden, 2001). Estima-se que cerca de 60\% dos fármacos com atividades antitumorais e antimicrobianas, já comercializados ou em fase de pesquisa clínica, sejam de origem natural (Shu, 1998).

As plantas medicinais desempenham, portanto, papel muito importante na medicina moderna. Primeiramente porque podem fornecer fármacos extremamente importantes, os quais dificilmente seriam obtidos via síntese química, como por exemplo, os alcalóides da Papaver somniferum e os glicosídeos cardiotônicos da Digitalis spp. Em segundo lugar, as fontes naturais fornecem compostos que podem ser levemente modificados, tornando-os mais eficazes ou menos tóxicos. Em terceiro lugar, os produtos naturais podem ser utilizados como protótipos para obtenção de fármacos com atividades terapêuticas semelhantes a dos compostos originais (Robbers, Speedie, Tyler, 1996).

Diante da grande importância dos medicamentos fitoterápicos, vários países da Europa estão intensificando esforços para unificar a legislação referente aos medicamentos fitoterápicos, amplamente comercializados nestes países (em especial na Alemanha e França). Por outro lado, nos Estados Unidos, as preparações à base de plantas são classificadas como suplementos nutricionais, não sendo necessário submeter dados de segurança e eficácia ao Food and Drug Administration (FDA) para a comercialização destes produtos.

No Brasil, a legislação para medicamentos fitoterápicos vem sofrendo modificações nos últimos anos. A Agência Nacional de Vigilância Sanitária (ANVISA) vem elaborando normas para a regulamentação destes medicamentos, desde a Portaria n. 6 de 1995 , que estabeleceu prazos para que as indústrias farmacêuticas apresentassem dados de eficácia e segurança dos medicamentos fitoterápicos, passando pela RDC n. 17 de 2000, e a Resolução RDC n. 48 de 16 de março de 2004, 
atualmente em vigor, que dispõe sobre o registro de medicamentos fitoterápicos.

Esta preocupação das autoridades regulatórias com a normatização dos medicamentos fitoterápicos propicia a avaliação de aspectos importantes, como a eficácia e segurança do uso destes medicamentos. O uso tradicional de diversas plantas medicinais baseado em conhecimentos populares, aliado à crença de que, por ser natural não causa reações adversas, fez com que poucas plantas medicinais fossem avaliadas através de estudos pré-clínicos e clínicos, a fim de comprovar sua eficácia e segurança.

Além disto, sabe-se que muitas plantas medicinais apresentam substâncias que podem desencadear reações adversas, seja por seus próprios componentes, seja pela presença de contaminantes ou adulterantes presentes nas preparações fitoterápicas, exigindo um rigoroso controle de qualidade desde o cultivo, coleta da planta, extração de seus constituintes, até a elaboração do medicamento final.

Assim, o objetivo do presente trabalho foi avaliar as informações sobre a toxicidade pré-clínica de dez plantas medicinais comercializadas como medicamentos fitoterápicos no Brasil junto aos principais bancos de dados e fontes públicas de informação.

\section{MATERIAL E MÉTODO}

\section{Material}

Para este estudo, foram selecionadas dez plantas medicinais comercializadas na forma de medicamentos fitoterápicos, os quais apresentaram volume de vendas e número de unidades vendidas significativos entre os anos de 1999 e 2002, segundo informações da IMS Health. As plantas selecionadas são as seguintes:

\section{Passiflora incarnata L. \\ 2. Ginkgo biloba L. \\ 3. Aesculus hippocastanum L. \\ 4. Plantago ovata Forsk. \\ 5. Panax ginseng C. A Meyer \\ 6. Piper methysticum G. Forst. \\ 7. Valeriana officinalis $\mathrm{L}$. \\ 8. Hypericum perforatum $\mathrm{L}$. \\ 9. Cimicifuga racemosa (L.) Nutt. \\ 10.Rhamnus purshiana D.C.}

\section{Método}

As dez plantas medicinais selecionadas foram avaliadas quanto aos aspectos toxicológicos nas bases de dados e fontes de referência disponíveis, apresentadas na Tabela I.

\section{RESULTADOS}

As Figuras 1 e 2 destacam, respectivamente, a evolução do volume de vendas e o faturamento dos medicamentos fitoterápicos à base das dez plantas medicinais selecionadas, no período 1999-2002.

As Tabelas II a XI apresentam informações toxicológicas obtidas junto às fontes de informações pesquisadas para as dez plantas selecionadas.

\section{DISCUSSÃO}

Para as dez plantas selecionadas neste trabalho, foram avaliadas as informações com relação à toxicidade aguda, subaguda e crônica, mutagenicidade e teratogenicidade em importantes fontes de informação, como The Complete German Commission E Monographs (Blumenthal, 1998); WHO Monographs on Selected Medicinal Plants (WHO, 1999 e 2002); Monographs on the medicinal Uses of Plant Drugs (ESCOP, 1999). Observamos, por exemplo, que, para Piper methysticum, as três fontes listadas acima não indicam quaisquer dados sobre estudos de toxicidade aguda, subaguda e crônica, o mesmo ocorrendo com Hypericum perforatum e Cimicifuga racemosa, embora as demais bases pesquisadas apresentem algumas informações.

Para Passiflora incarnata, a disponibilidade de informações sobre sua toxicidade é limitada e apoiada no seu uso bem-estabelecido na medicina tradicional, conforme a base de dados Thomson MICROMEDEX. Apesar dessa relativa escassez, uma das referências (Fisher, Purcell, Le Couteur, 2000) relata eventos adversos cardiovasculares e gastrintestinais após seu uso em doses terapêuticas por uma mulher jovem, os quais poderiam estar relacionados aos alcalóides e flavonóides presentes na formulação. A Passiflora incarnata é utilizada em combinação com Valeriana officinalis e Melissa officinalis, chamado "Chá Sedativo", aprovado na Alemanha pela Comissão "E".

Já com relação a Ginkgo biloba, mais de 400 estudos foram conduzidos com os extratos padronizados de suas folhas nos últimos 30 anos e somente os extratos padronizados comprovam os efeitos terapêuticos estudados. Outras formas de utilização, como as folhas frescas, ou os extratos em baixas concentrações, não produzem os efeitos desejados (Blumenthal, 2000). O primeiro extrato desenvolvido foi o EGb 761, por W.Schwabe Co., na Alemanha, o qual foi intensivamente estudado quanto aos aspectos químicos, farmacológicos e toxicológicos. Os ginkgolídeos, em especial o ginkgolídeo B, podem agir 


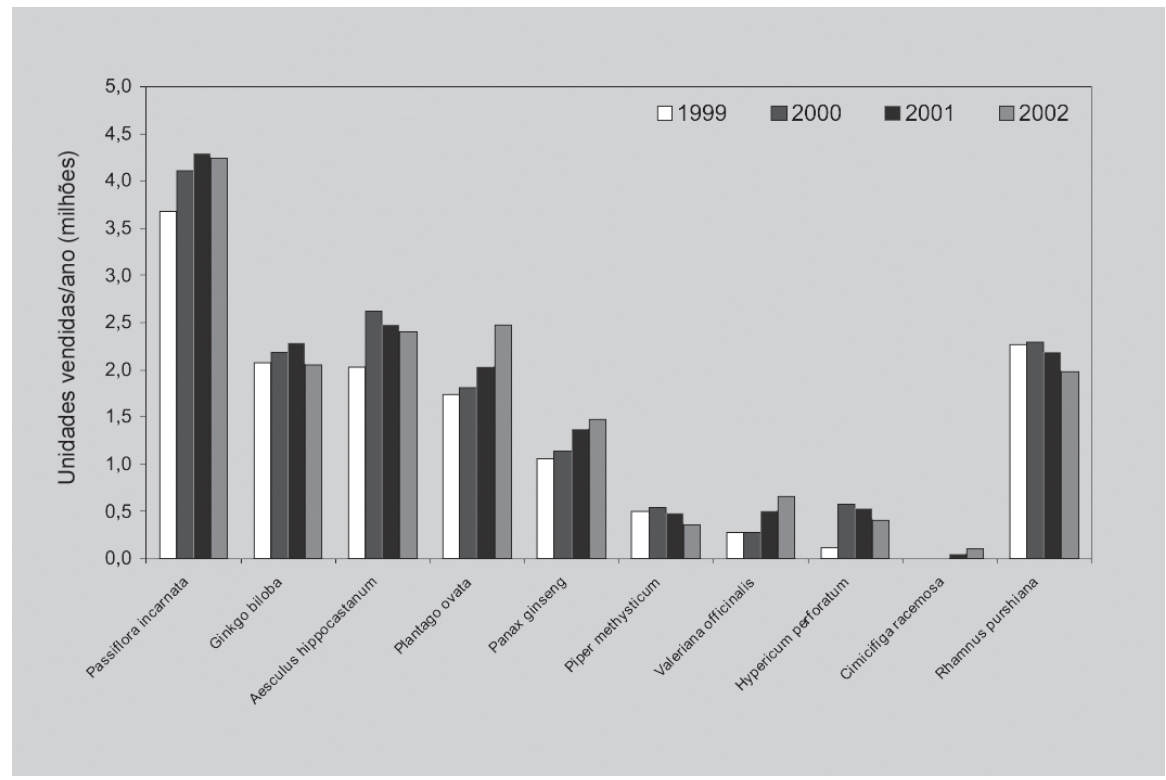

FIGURA 1 - Evolução da quantidade vendida dos medicamentos fitoterápicos à base das dez plantas medicinais selecionadas. Brasil,1999-2002. Fonte: IMS Health - dados pesquisados em outubro 2003.

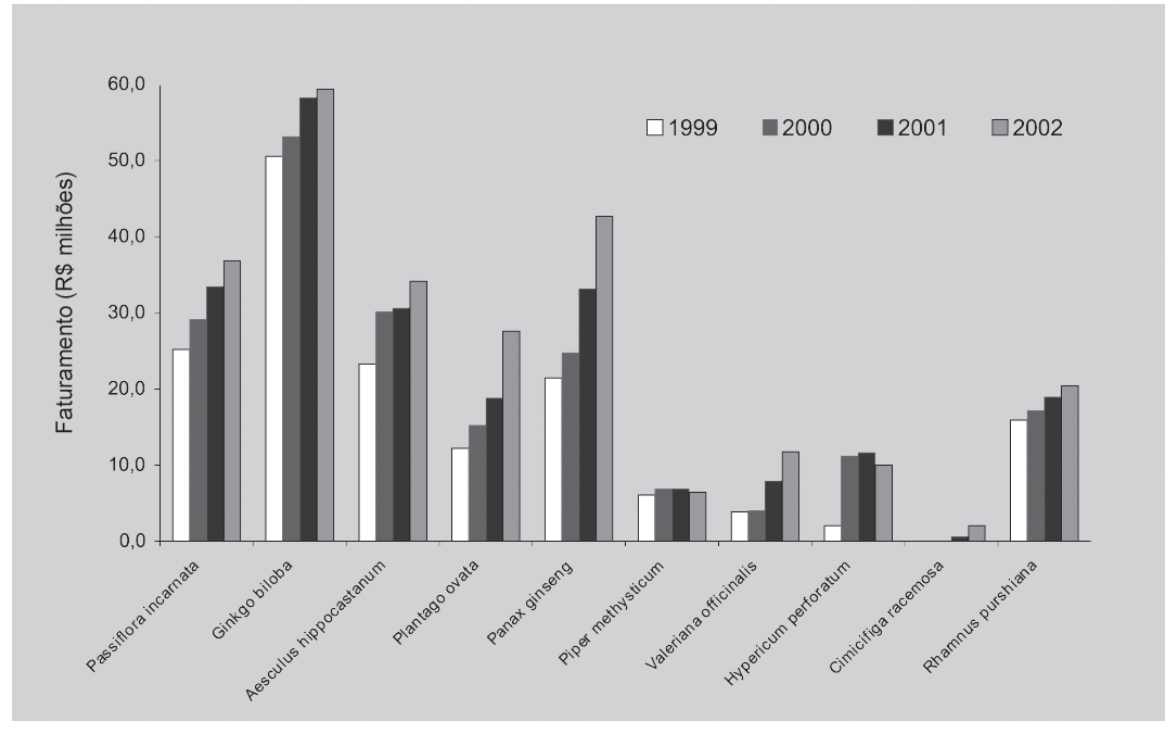

FIGURA 2 - Evolução do faturamento dos medicamentos fitoterápicos à base das dez plantas medicinais selecionadas. Brasil, 1999-2002. Fonte: IMS Health - dados pesquisados em outubro 2003.

como um potente inibidor do fator ativador de plaquetas e seu uso crônico pode estar associado com o aumento no tempo de sangramento e com o risco de hemorragia espontânea. Glicosídeos cianogênicos podem ser encontrados na planta, mas sua atividade biológica não foi estudada (WHO, 1999). As sementes, caules e folhas contém 4'-Ometilpiroxidina, que causa sintomas de deficiência da vitamina $\mathrm{B}_{6}$, incluindo convulsões (Mils, Bone, 2000).

Outras plantas também foram avaliadas em estudos clínicos. Em grande estudo com indivíduos expostos a 8 espécies do gênero Aesculus, 3099 casos foram analisados de 1985 a 1994. Cerca de 50\% das exposições ocorreram com crianças entre 0 e 5 anos de idade: em 77\% (2374) dos casos não foram detectados quaisquer efeitos tóxicos; em 11,5\% (356) dos casos ocorreram efeitos mínimos a moderados. Nos demais $11,5 \%$ (359) dos casos, os efeitos foram classificados como toxicidade potencialmente desconhecida. Matyunas et al. (1997) concluíram que a maioria das exposições não resultou em efeitos tóxicos; nenhuma toxicidade grave foi relatada segundo dados de Thomson MICROMEDEX. 


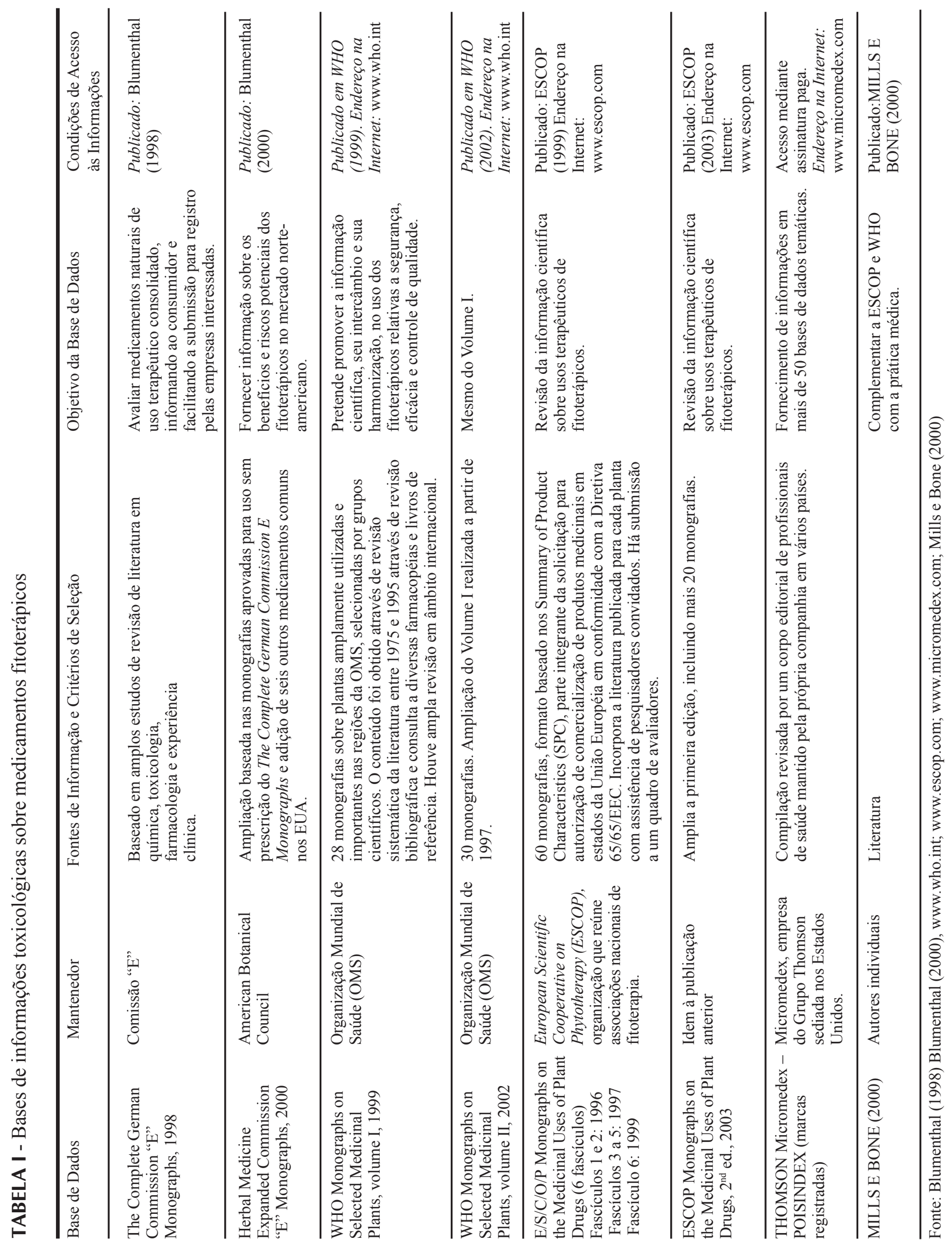




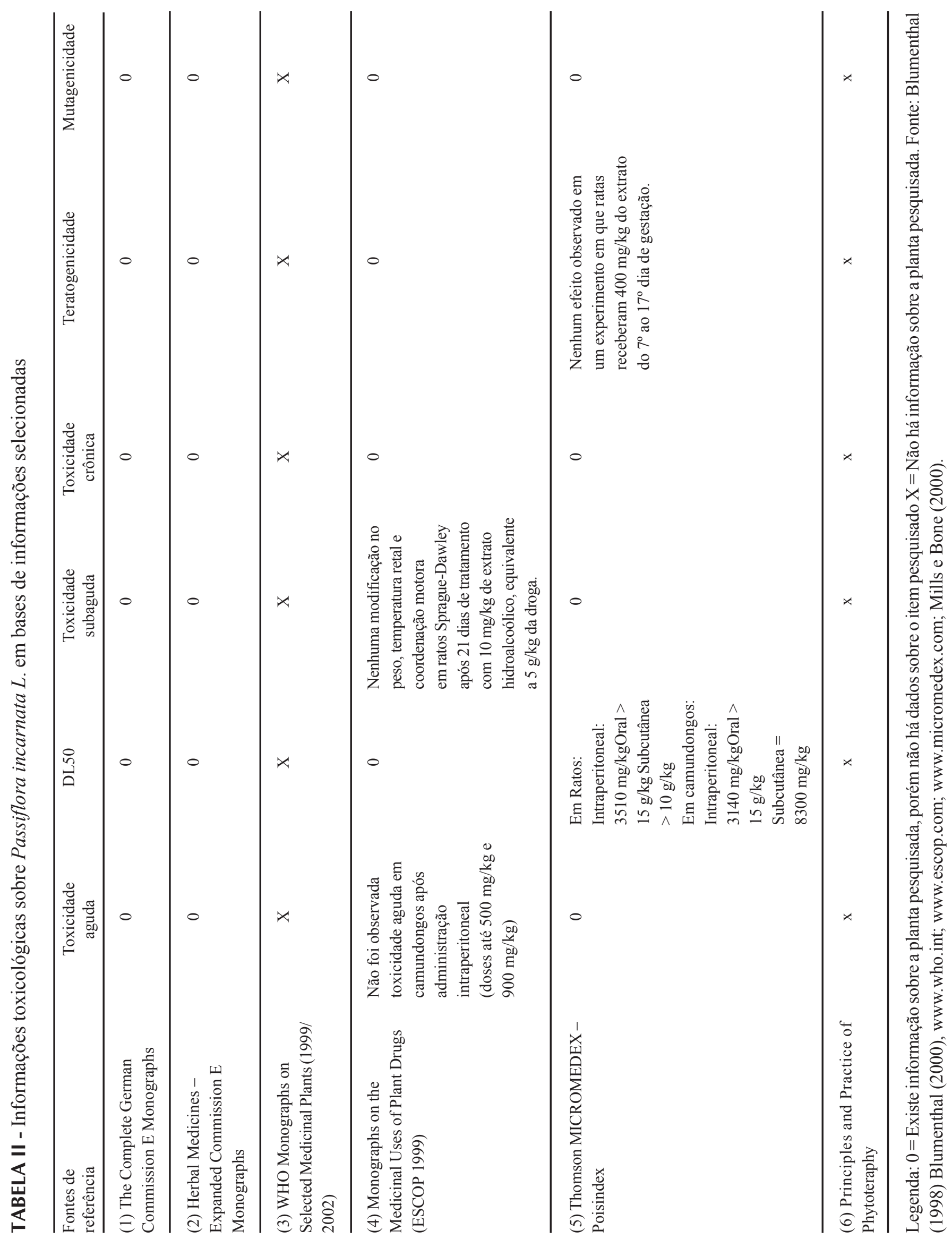




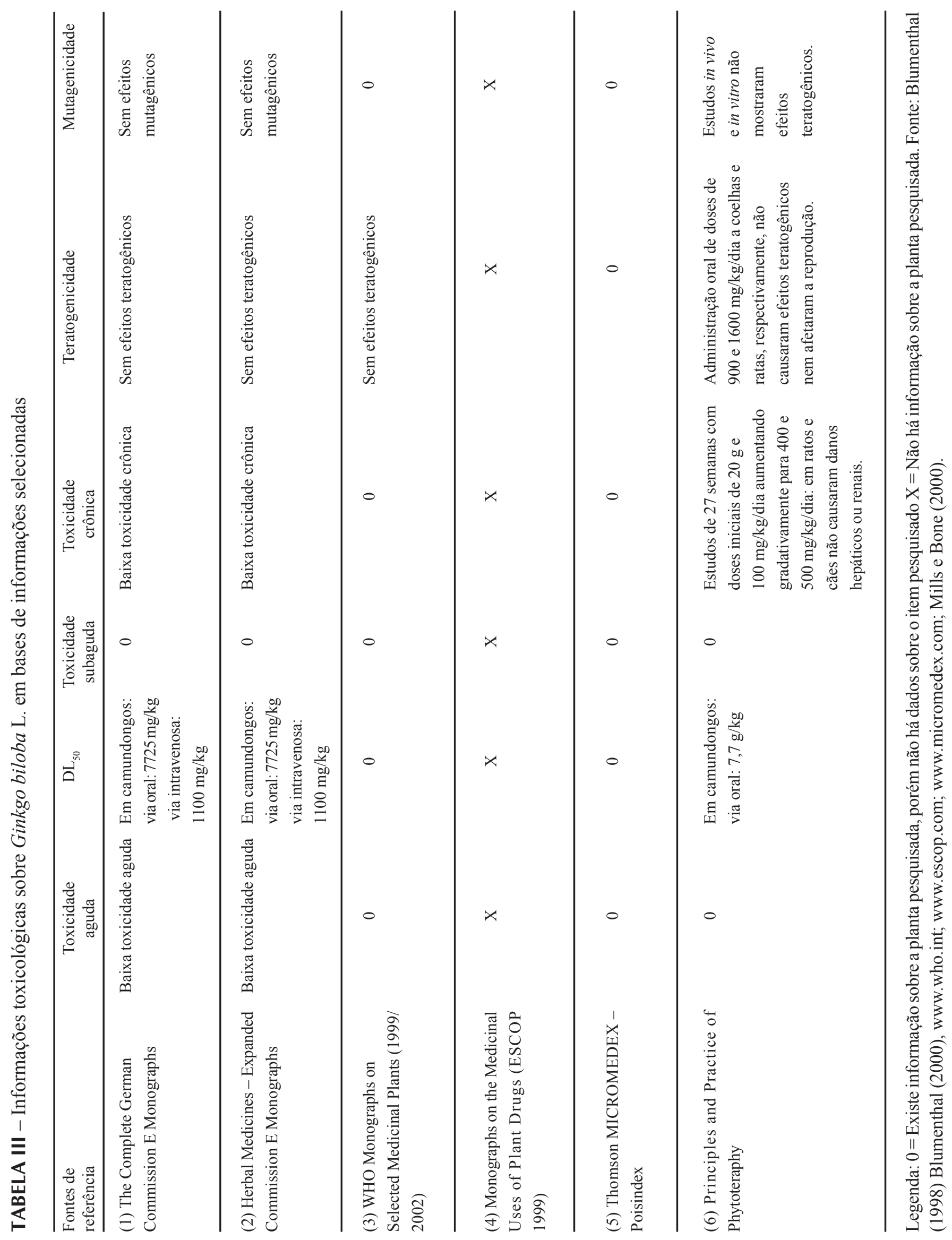




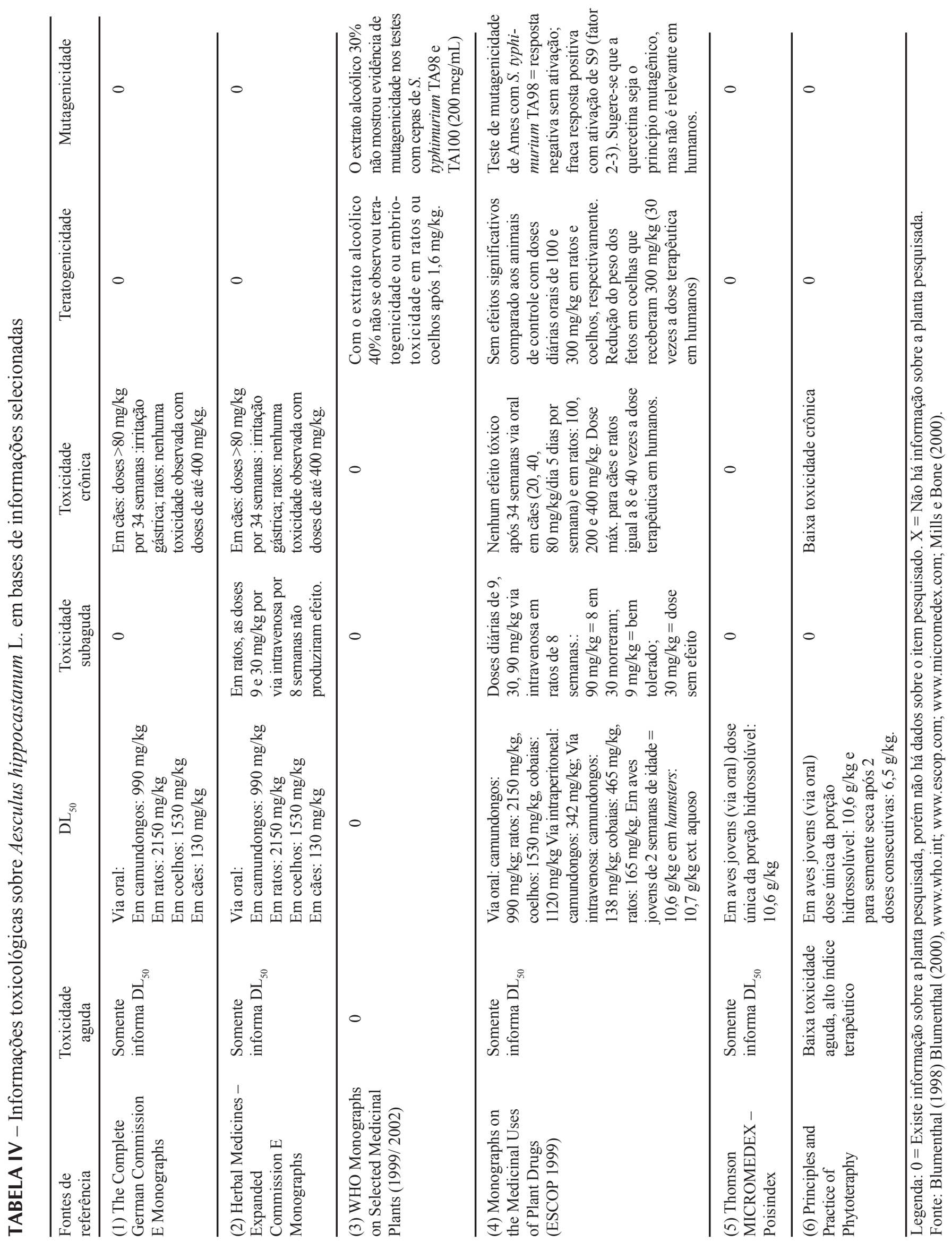


Informações toxicológicas de alguns fitoterápicos utilizados no Brasil

297

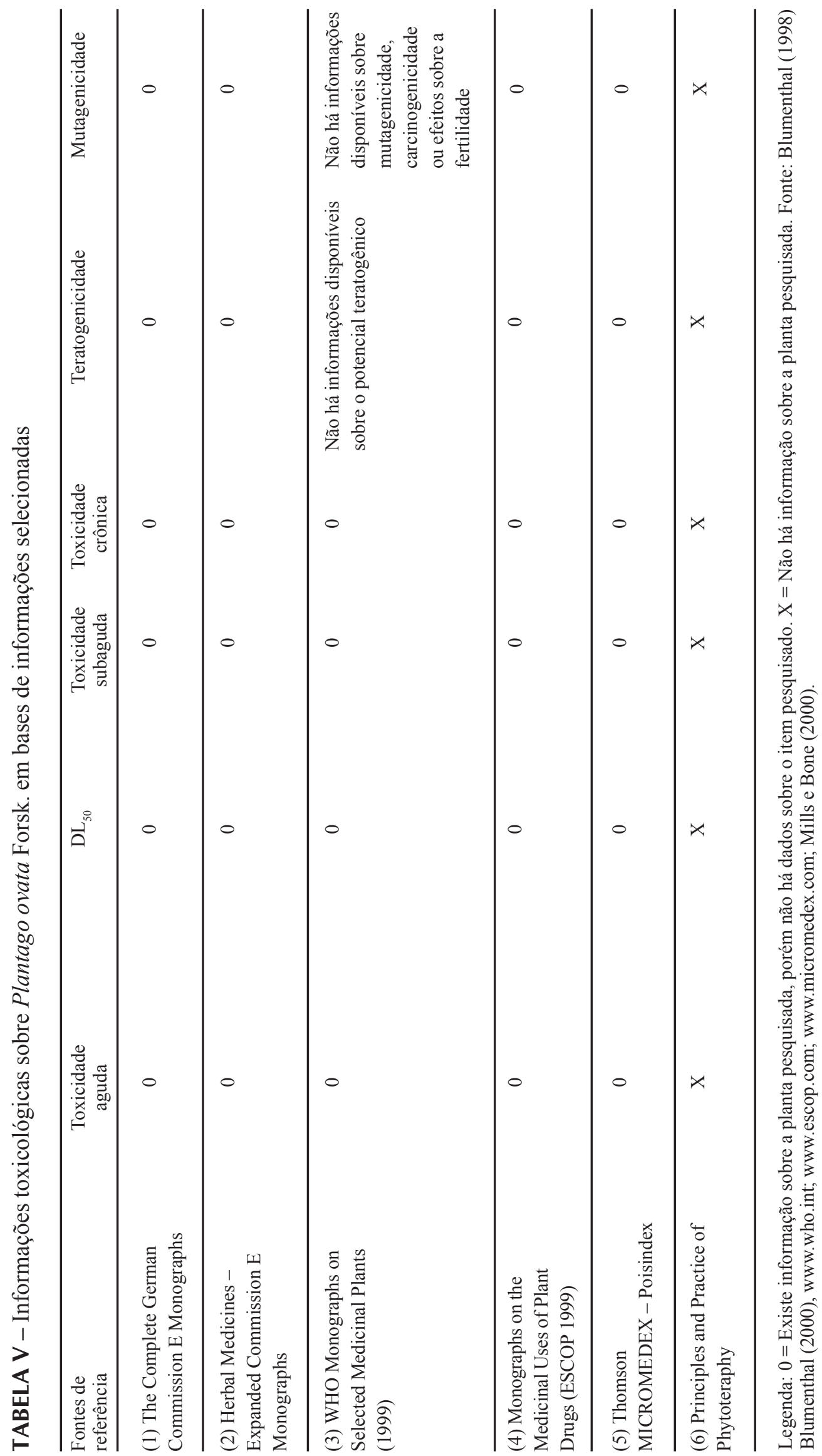




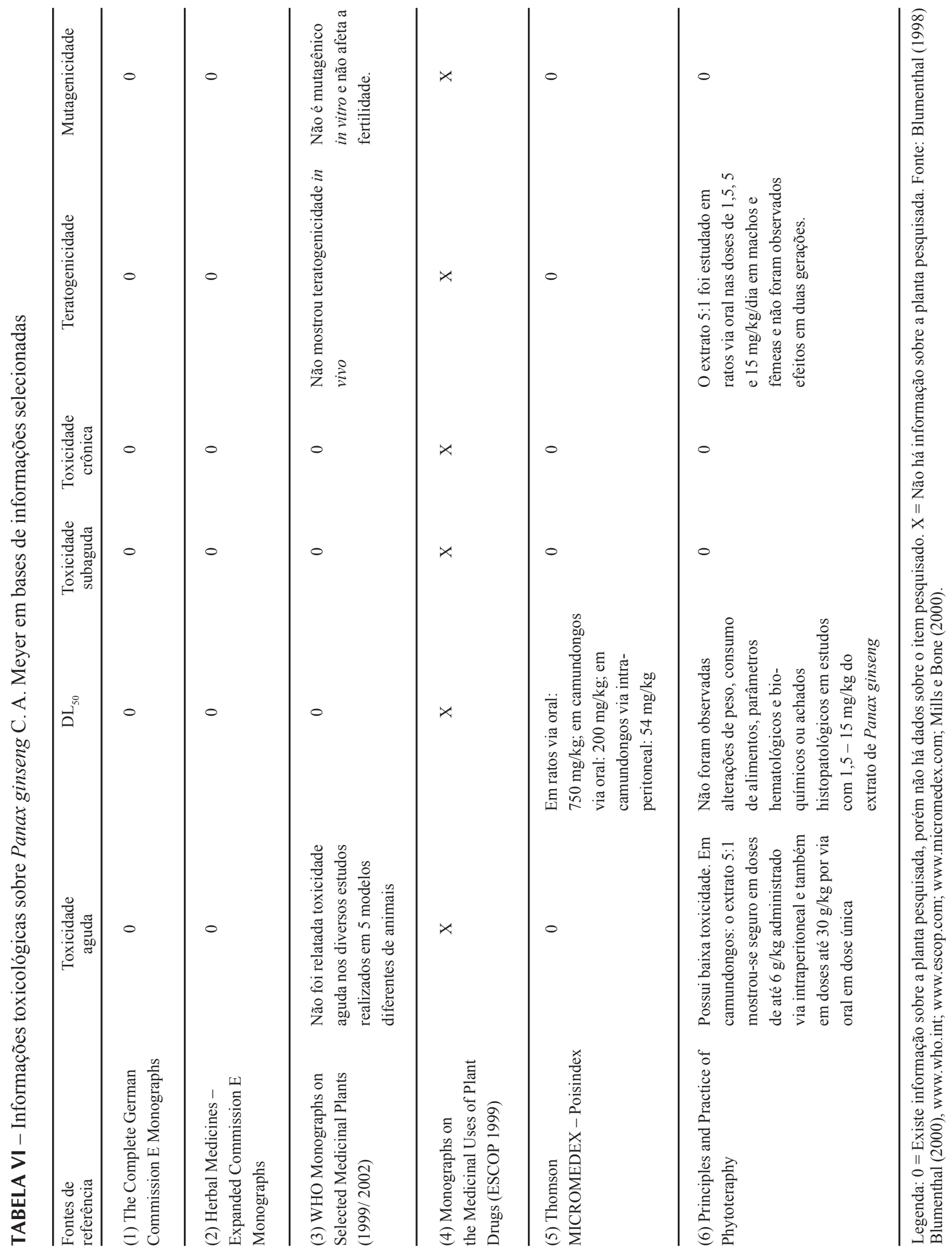


Informações toxicológicas de alguns fitoterápicos utilizados no Brasil

299

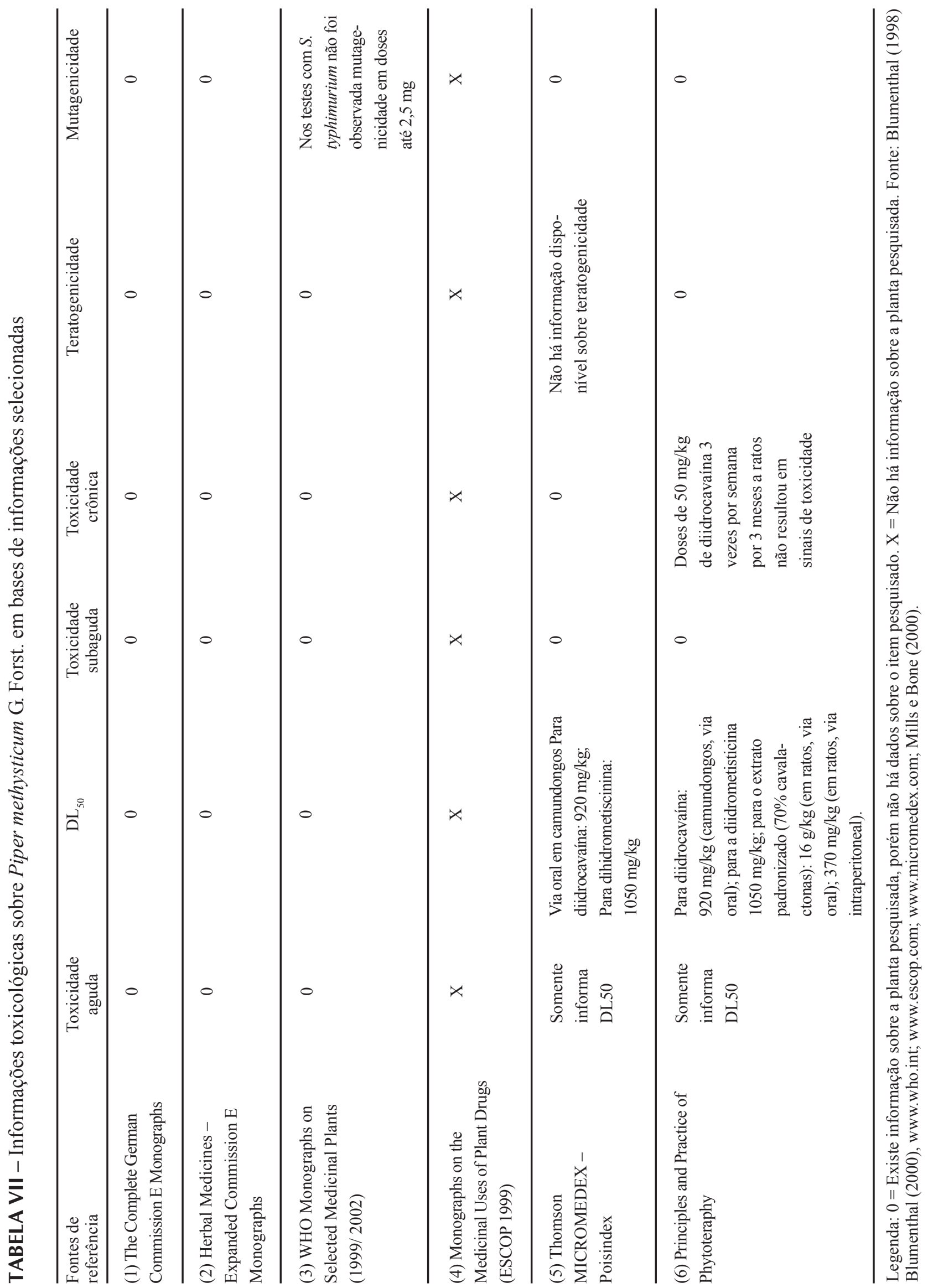




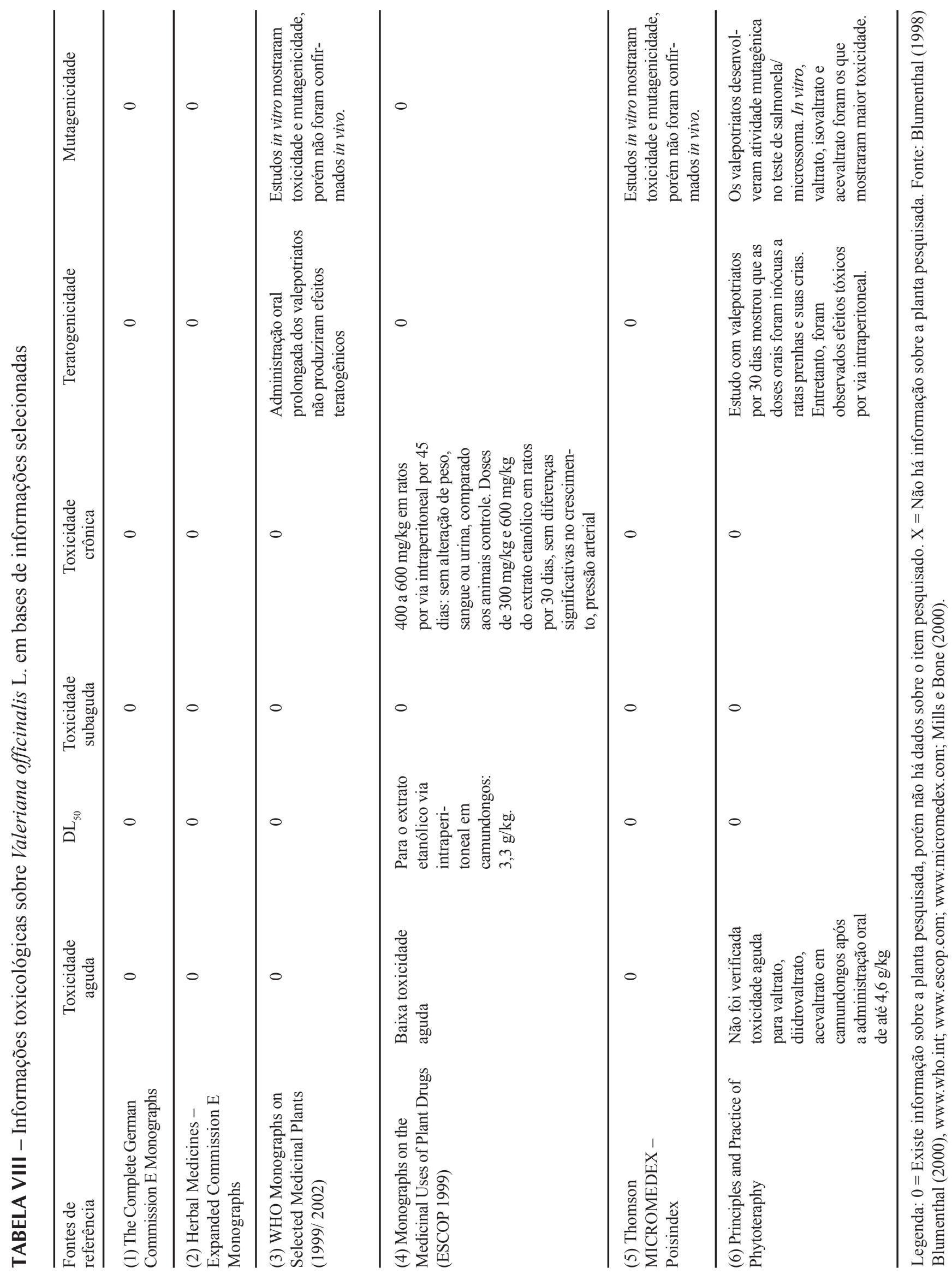




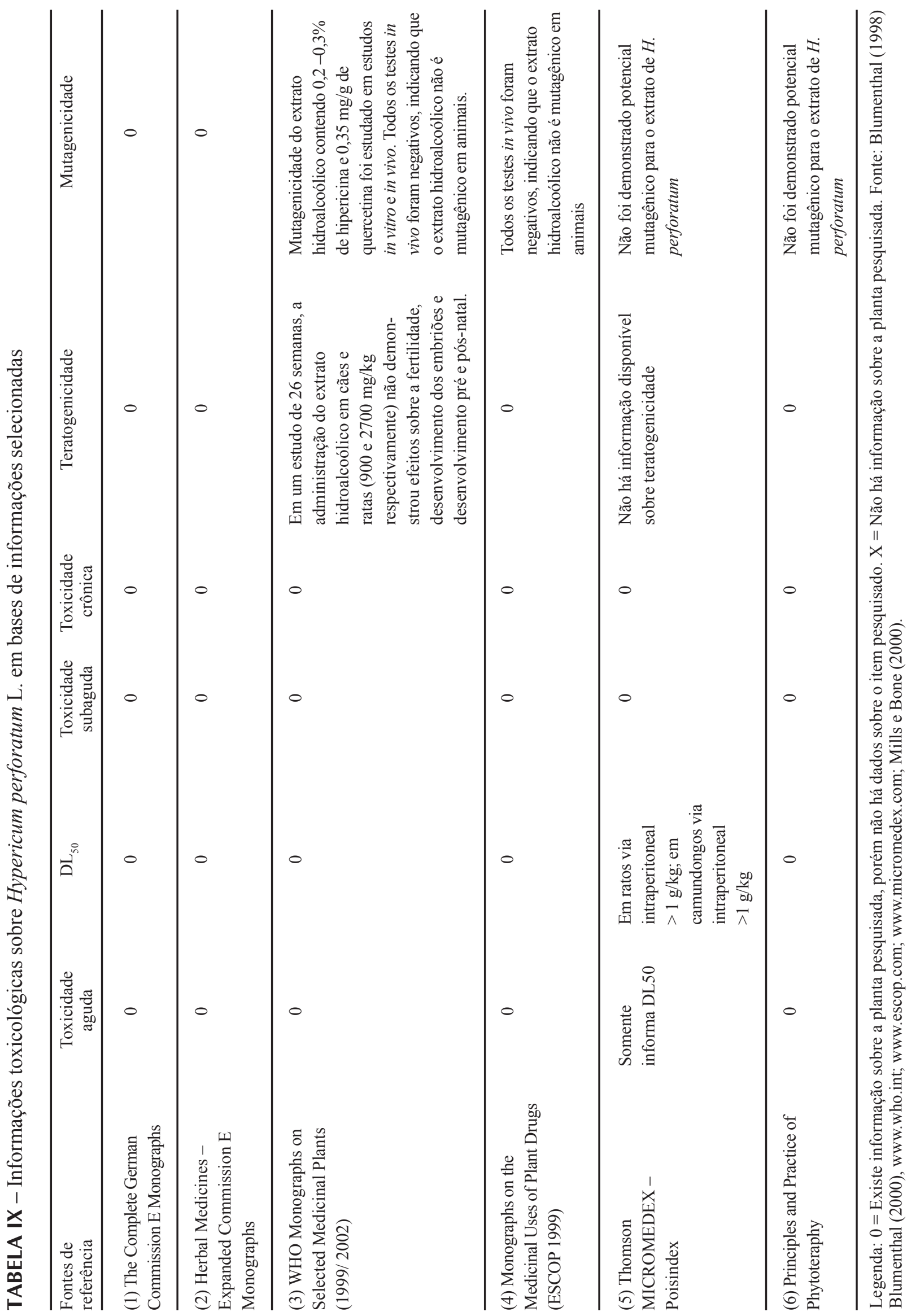




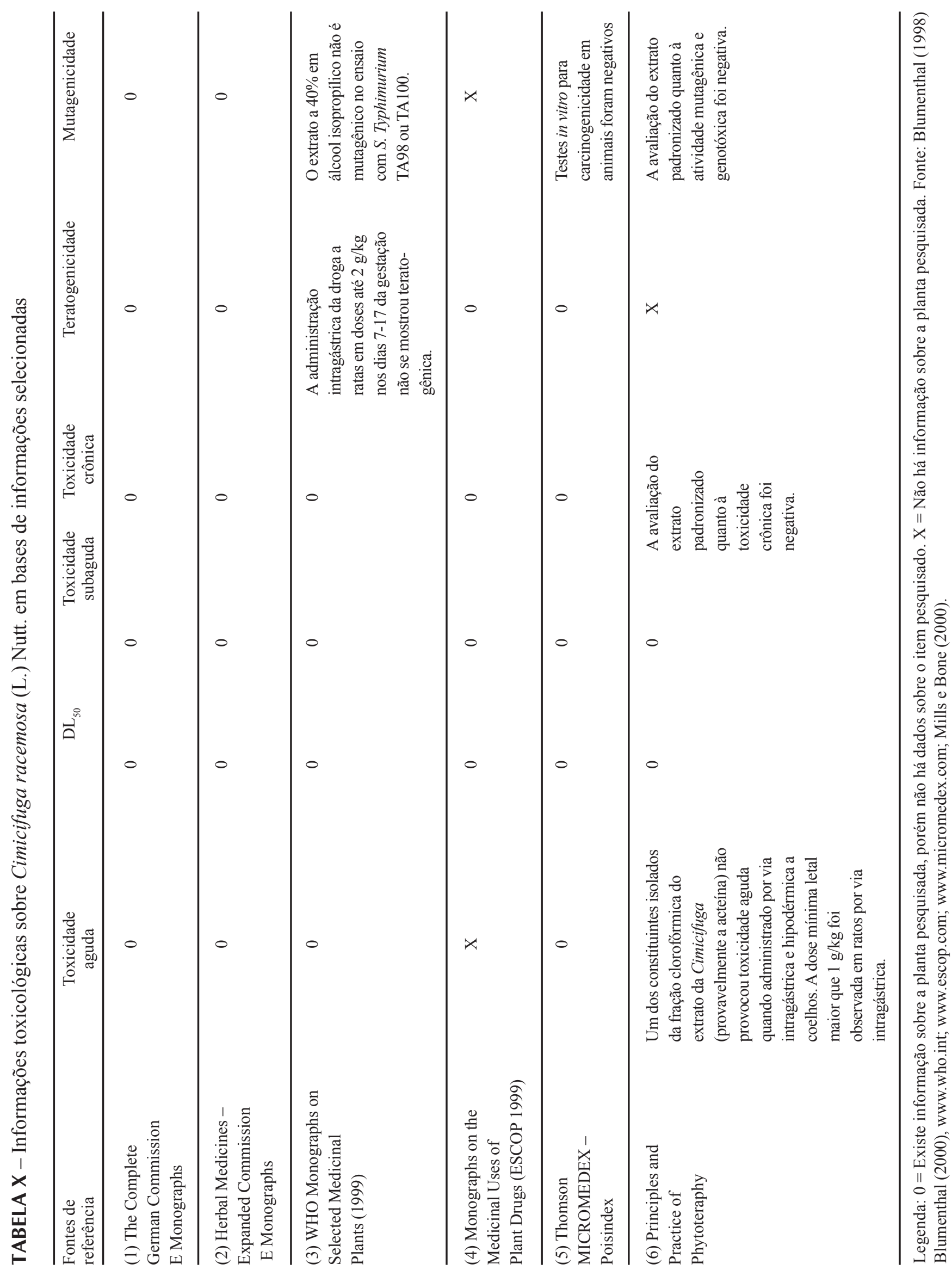




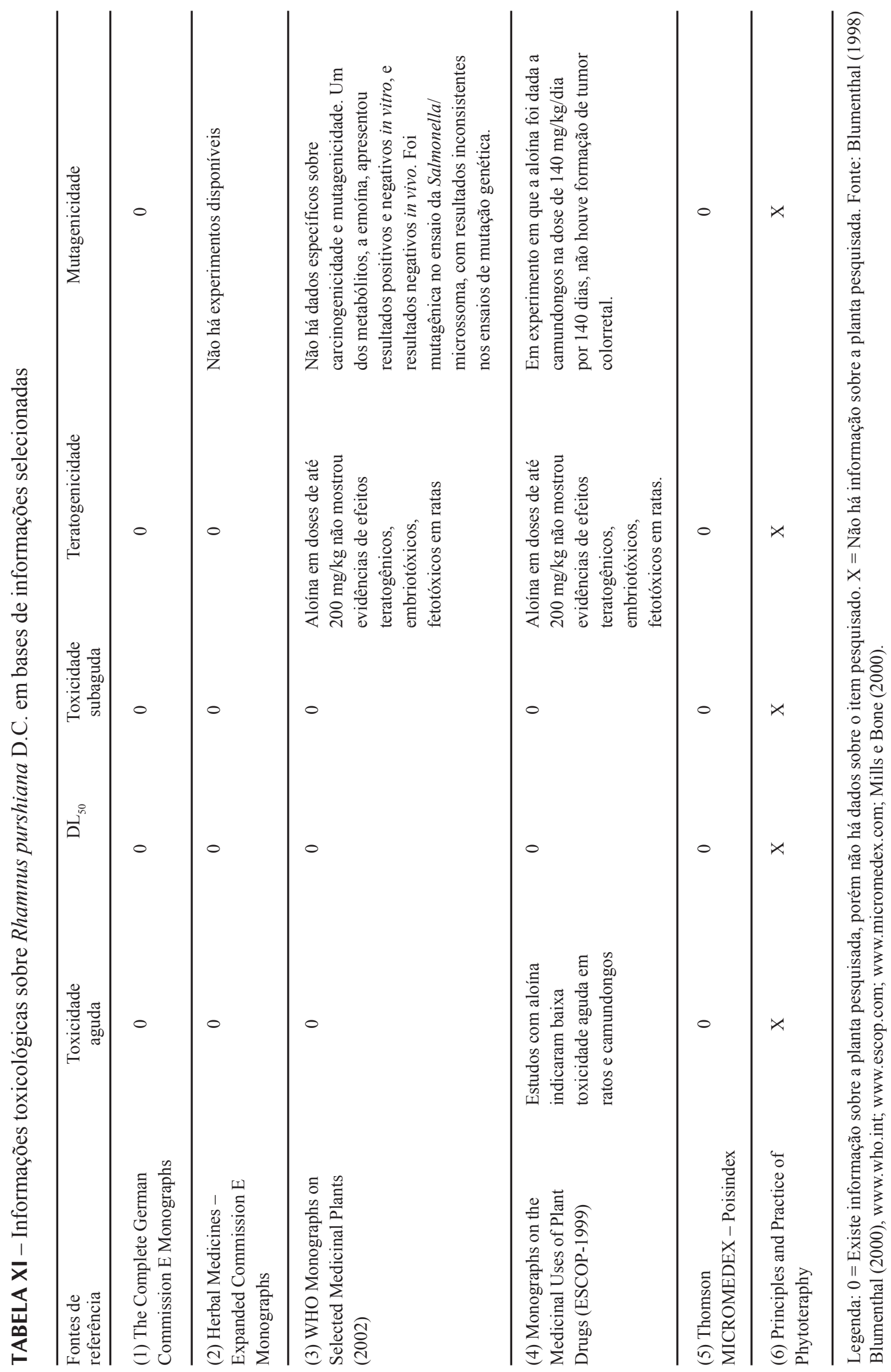


As fibras de Plantago ovata são também utilizadas há muito tempo na medicina tradicional na Ásia, Europa e América do Norte. O grande risco associado ao seu uso está na ingestão insuficiente de líquidos com o medicamento, o que pode causar obstrução esofágica especialmente em pessoas idosas, que apresentam motilidade gastrintestinal prejudicada, segundo dados de Thomsom MICROMEDEX.

Outra planta utilizada tradicionalmente é o Panax ginseng, usada extensivamente pela Medicina Tradicional Chinesa na forma de chás e outras formas líquidas ou sólidas prescritas por acupunturistas e médicos naturalistas. A administração crônica de Panax ginseng foi associada à ocorrência de sangramento vaginal, mastalgia, alteração do estado mental. Também foi relatada síndrome de abuso por ingestão crônica de Panax ginseng, podendo ocorrer hipertensão, nervosismo, insônia, erupções cutâneas e diarréia matinal segundo Thomsom MICROMEDEX.

Em relação ao Hypericum perforatum, foram relatados efeitos adversos importantes. Nas bases de dados WHO Monographs e ESCOP há artigos que descrevem a fototoxicidade da hipericina, um dos principais constituintes com atividade antidepressiva. Em estudos com animais foi observada reação de fotossensibilidade em bovinos, ovinos e eqüinos, principalmente os de pele mais clara, após a ingestão de grandes quantidades de Hypericum perforatum (cerca de trinta vezes a dose preconizada em humanos). Em estudos clínicos também foi demonstrada fotossensibilidade em indivíduos sensíveis que foram expostos a radiações ultravioleta $\mathrm{A}$ e B.

Com relação ao Piper methysticum, seu uso foi relatado desde 1768, tendo sido utilizado em rituais das comunidades das ilhas do Pacífico Sul na forma de bebida, a qual causava sensação adstringente e sedativa, seguido por relaxamento, com diminuição da fadiga e ansiedade. Há relatos de que o consumo excessivo desta bebida em doses elevadas por essas comunidades resultou em má nutrição, perda de peso, disfunções hepáticas e renais, entre outros. Entretanto, não se sabe ao certo se estes efeitos foram provocados somente pelo Piper methysticum, ou se foram potencializados pelo álcool (Mills, Bone, 2000).

Como foi observado nas referências pesquisadas, o uso tradicional das plantas medicinais não garante a segurança do medicamento fitoterápico, sendo necessário realizar ensaios pré-clínicos e clínicos. Os testes para avaliação de toxicidade das substâncias químicas estão bem detalhados segundo os protocolos sugeridos pela OECD (1996). No Brasil, a Resolução RDC n. 48 e a Resolução - RE n. 90, publicadas em 16 de março de 2004 , tratam do registro dos medicamentos fitoterápicos e apresentam um guia para a realização de estudos de toxicidade pré-clínica de fitoterápicos, respectivamente. A resolução RE n. 90 foi elaborada em conformidade com as normas da Organização Mundial de Saúde (OMS), e recomenda estudos de toxicidade aguda e de doses repetidas.

Com relação ao estudo de toxicidade de doses repetidas, a OMS e a RE n. 90 de 2004 sugerem a utilização de pelo menos duas espécies roedoras e não-roedoras, sendo porém o período de administração do produto nos animais baseado no período proposto para a utilização terapêutica da substância, diferentemente das normas da OECD, que especificam o período de administração em 28 e 90 dias para espécies roedoras e 90 dias para espécies não-roedoras. Além disto, nem a OMS e nem a RE n. 90 de 2004, informam a concentração máxima da substância-teste que pode ser administrada nos ensaios de toxicidade aguda e de doses repetidas.

Além dos ensaios de toxicidade aguda e de doses repetidas, a RE n. 90 de 2004 também sugere estudos de genotoxicidade, quando houver indicação de uso contínuo ou prolongado do medicamento em humanos, e a avaliação toxicológica de fitoterápicos de uso tópico, porém não detalha os métodos para a realização destes testes. Assim, levando-se em consideração o estágio de desenvolvimento que já foi atingido pelos protocolos da OECD, os quais especificam somente os testes de toxicidade para substâncias químicas, ainda há dificuldades em se estabelecer os testes mais adequados para os fitoterápicos, principalmente diante da dificuldade de padronização das preparações fitoterápicas.

Apesar dos avanços já conquistados, a regulamentação dos medicamentos fitoterápicos permanece como uma questão em aberto, mesmo entre os países da União Européia, em especial na Alemanha e na França onde os fitoterápicos são mais comercializados. Enquanto toda a legislação para os medicamentos de fármacos sintéticos encontra-se bem estabelecida, os medicamentos fitoterápicos ainda carecem de maior esforço regulatório.

Em contrapartida, os Estados Unidos consideram os medicamentos fitoterápicos como suplementos nutricionais, não sendo necessário submeter dados de segurança e eficácia ao FDA para sua comercialização. Assim, o grande problema com relação aos suplementos nutricionais é que não há controle de qualidade destes produtos, logo não há como provar que as substâncias e as quantidades declaradas nos rótulos estão presentes, bem como não há controle sobre outras substâncias como fármacos 
sintéticos ou outros contaminantes, que podem estar presentes na formulação, os quais podem resultar em interações indesejáveis.

\section{CONCLUSÕES}

A pesquisa apresentada neste trabalho mostrou que poucos dados sobre a toxicidade pré-clínica das dez plantas avaliadas estão descritos nas fontes de informações utilizadas. As monografias da Organização Mundial da Saúde e da Comissão "E" contemplam as dez plantas avaliadas, porém apresentam poucos dados sobre as informações pesquisadas. De maneira geral, os experimentos em animais revelam baixa toxicidade aguda, subaguda e crônica, bem como não demonstraram atividade mutagênica ou teratogênica para as dez plantas avaliadas.

\section{ABSTRACT \\ Toxicological information of some herbal medicines used in Brazil}

Herbal medicines have been used with therapeutical purposes for thousands of years. Its popular use has been transmitted across generations and is described in the pharmacopeas. Since the development of organic chemistry, it became possible to obtain pure substances by isolating active substances from plants, among them, digoxine and morphine, resulting in lack of interest for research of substances originated from vegetals. However, from the 1980's onwards, new methods for isolating active substances were developed and it became possible to identify substances in complex samples such as vegetal extracts, restoring the interest for vegetally-originated compounds that could be used as prototypes for the development of new drugs. Nowadays, in spite of the growing importance of herbal medicines, relatively few studies have been performed to prove efficacy and safety, while many plants are still used on the basis of popular well-established use. The objective of this paper is to assess the availability of preclinical toxicity data for ten medicinal plants marketed as herbal medicines in Brazil, in major databases and public sources of information. Few data were found regarding pre-clinical toxicity on the ten medicinal plants surveyed, but, in general, experiments in animals showed low acute, sub-acute and chronical toxicity and did not show mutagenic or teratogenic toxicity.

UNITERMS: Toxicological information. Herbal medicines. Medicinal plants. Pre-clinical toxicity.

\section{REFERÊNCIAS BIBLIOGRÁFICAS}

ASCHWANDEN, C. Herbs for health, but how safe are they? Bull. W. H. O., Geneva, v.79, n.7, p.691-692, 2001. Disponível em: http://www.who.int/bulletin. Acesso em: 09 jan. 04.

BLUMENTHAL, M., ed. Herbal medicine: expanded commission E monographs. Newton: Integrative Medicine Communication/American Botanical Council, 2000.519p.

BLUMENTHAL, M.; BUSSE, W.R.; KLEIN, S., eds. The complete German Commission E monographs: therapeutic guides to herbal medicines. Austin: American Botanical Council, 1998. 685p.

BRASIL. Ministério da Saúde. Agência Nacional de Vigilância Sanitária. Legislação. Sistema de Legislação em Vigilância Sanitária (VISALEIS). Resolução RDC n. 48 de 16 de março de 2004. Disponível em: http://e-legis.bvs.br/leisref/ public/search.php. Acesso em: 25 jun. 2004.

BRASIL. Ministério da Saúde. Agência Nacional de Vigilância Sanitária. Legislação. Sistema de Legislação em Vigilância Sanitária (VISALEIS). Resolução RE n. 90 de 16 de março de 2004. Disponível em: http://e-legis.bvs.br/leisref/ public/search.php. Acesso em: 25 jun. 2004.

BRASIL. Ministério da Saúde. Agência Nacional de Vigilância Sanitária. Consultas Públicas. Áreas de Atuação. Medicamentos. Legislação. Resoluções. Resolução - RDC n ${ }^{\circ} .17$ de 24 de fevereiro de 2000. Disponível em http://www.anvisa.gov.br/medicamentos/ legis/resol.htm . Acesso em 24 jan 2004.

ELVIN-LEWIS, Memory. Should we be concerned about herbal medicines? J. Ethnopharmacol., Amsterdam, v.75, p.141-164, 2001.

EUROPEAN SCIENTIFIC COOPERATIVE ON PHYTOTHERAPY. Monographs on the medicinal uses of plant drugs. Exeter: ESCOP, 1996. 1v.

FISHER, A.A.; PURCELL, P.; LE COUTEUR, D.G. Toxicity of Passiflora incarnata L. Clin. Toxicol., New York, v.38, n.1, p.63-66, 2000.

IMS HEALTH. Intercontinental Marketing Services. Company Information. Disponível em: www.imshealth.com. Acesso em: 30 abr. 2005. 
KO, R.J. Causes, epidemiology, and clinical evaluation of suspected herbal poisoning. Clin. Toxicol., New York, v.37, n.6, p.697-708, 1999.

MATYUNAS, N.; KRENZELOK, E.; JACOBSEN, T.; et al. Horse chestnut (aesculus spp.) ingestion in the United States: 1985-1994. J. Toxicol. - Clinical Toxicol., v.35, p. 527, 1997.

MILlS, S., BONE, K. Principals and practice of phytotherapy: modern herbal medicine. Edinburgh, Londres: Churchill Livingstone, 2000. 643p.

OECD - ORGANIZATION FOR ECONOMIC COOPERATION AND DEVELOPMENT. OECD guidelines for testing of chemicals. Paris: OECD, 1996.

RATES, S.M.K. Plants as source of drugs. Toxicon, Amsterdam, v.39, p.603-613, 2001.

ROBBERS, J.E.; SPEEDIE, M.K.; TYLER, V.E. Pharmacognosy and pharmacobiotechnology. Baltimore: Willians \& Wilkins, 1996. p.1-14.

SCHENKEL, E.P.; GOSMANN, G.; PETROVICK, P.R. Produtos de origem vegetal e o desenvolvimento de medicamentos. In: SIMÕES, C.M.O.; SCHENKEL, E.P.; GOSMANN, G.M.; MELLO, J.C.P.; MENTZ, L.A.; PETROVICK, P.R., orgs. Farmacognosia: da planta ao medicamento. 2.ed. Florianópolis. UFSC; Porto Alegre: UFRGS, 2000. cap.15, p.291-320.
SCHULZ, V.; HÄNSEL, R.; TYLER, V.E. Medicinal plants, phytomedicines, and phytotherapy. In: Rational phytotherapy: a physician's guide to herbal medicine. 4.ed. New York, Berlin: Springer, 2001. cap.1, p.1-39.

SHU, Y.-Z. Recent natural products based drug development: a pharmaceutical industry perspective. J. Nat. Prod., Columbus, v.61, p.1053-1071, 1998.

TUROLLA, M. S. R. Avaliação dos aspectos toxicológicos dos fitoterápicos: um estudo comparativo. São Paulo, 2004. 131 p. (Dissertação de Mestrado. Faculdade de Ciências Farmacêuticas. Universidade de São Paulo).

TYLER, V.E. Natural products and medicine: an overview. In: BALICK, M.J.; ELISABETSKY, E.; LAIRD, S.A., eds. Medicinal resources of the tropical forest, biodiversity and its importance to human health. New York: Columbia University Press, 1996. p.3-10. (Biology and resource management series).

WORLD HEALTH ORGANIZATION. WHO monographs on selected medicinal plants. Geneva: Organização Mundial de Saúde, 1999. 2v.

Recebido para publicação em 04 de maio de 2005. Aceito para publicação em 24 de novembro de 2005. 